\title{
Global Impact Risk of Known Asteroids
}

\author{
Clemens Rumpf, University of Southampton, Faculty for Engineering and the Environment, SO171BJ, Southampton, \\ UK, c.rumpf@soton.ac.uk
}

Hugh G. Lewis, University of Southampton, Faculty for Engineering and the Environment, SO171BJ, Southampton, UK, h.g.lewis@soton.ac.uk

Peter M. Atkinson ${ }^{123}$, Lancaster University, Faculty of Science and Technology, LA14YR, Lancaster, UK, pma@lancaster.ac.uk

Asteroids that could collide with the Earth are listed on the publicly available Near Earth Object (NEO) hazard web sites maintained by the National Aeronautics and Space Administration (NASA) and the European Space Agency (ESA). The risk of 69 potentially threatening NEOs that produce 261 dynamically distinct impact instances, or Virtual Impactors (VIs), has been calculated using the Asteroid Risk Mitigation and Optimization Research (ARMOR) tool. ARMOR calculates the impact risk in terms of expected casualties based on three factors: impact probability, exposure and vulnerability. First, the impact probability of each VI is projected onto the surface of the Earth as a spatial probability distribution. The projection considers orbit solution accuracy and the global impact probability. Second, the global population distribution is introduced and represents the exposure to the hazard. Finally, the vulnerability of the population to the physical impact effects produced by a colliding asteroid is calculated. Impact effects are calculated based on asteroid size, impact speed and impact angle and the effects are: crater formation, thermal radiation, seismic shaking, overpressure shock wave, strong winds and the deposition of an ejecta blanket. Population vulnerability is determined based on the severity of the impact effects at a given distance from the impact site. Factoring together impact probability, exposure and vulnerability allows calculation of the risk for each VI as well as the combined risk of the 69 asteroids. To account for the uncertainty in the impact effect models, ARMOR produces three scenarios that represent the least harmful, the expected and the worst case outcomes. Because the risk calculation is dependent on the current impact probability, the risk calculation is subject to significant variability based on the availability of new asteroid observations. The calculated risk expresses the current best estimate of expected casualties that are associated with each asteroid. The method has the potential to form the basis of a new impact hazard threat scale similar to the Torino or Palermo scale. The results are presented in the form of global spatial risk distributions and as quantitative analysis.

\section{TABLE OF CONTENTS}

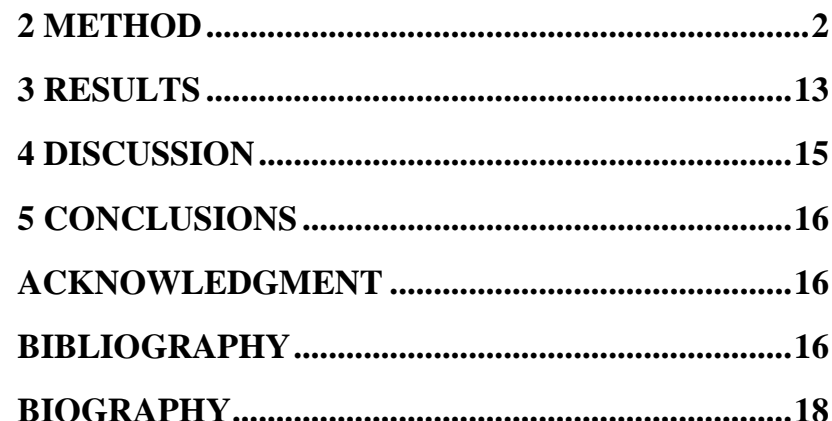

\section{INTRODUCTION}

Earth has collided with asteroids since it was a planetesimal and this process continues albeit at a lower rate [1]; it is a natural phenomenon. Asteroid impacts have been responsible for at least two major disruptions in the evolution of life [2], [3] and today, they remain a potential hazard for the human population [4], [5]. Surveys scan the sky for asteroids in an effort to discover as many as possible and to calculate their orbits [6]. Based on the propagation of orbits, those asteroids are identified that potentially impact the Earth in the future. The European Space Agency (ESA) and the National Aeronautics and Space Administration (NASA), perform the collision detection using automated systems and the results are

\footnotetext{
${ }^{1}$ University of Utrecht, Faculty of Geosciences, Heidelberglaan 2, 3584CS, Utrecht, The Netherlands

2 University of Southampton, Geography and Environment, SO171BJ, Southampton, UK

${ }^{3}$ Queen's University Belfast, School of Geography, Archaeology and Palaeoecology, BT71NN, Belfast, UK 
published on their respective Near Earth Object (NEO) webpages [7], [8]. At the University of Southampton, the Asteroid Risk Mitigation Optimization and Research (ARMOR) tool is under development with the objective of helping to analyse the threat posed by discovered asteroids. ARMOR projects the potential impact locations of an asteroid onto the surface of the Earth as its impact probability distribution. Furthermore, ARMOR calculates impact effects and determines the lethally affected population in the impact affected area. Consequently, ARMOR allows to calculate the risk of known asteroids in terms of expected casualties. For this paper, the risk of 69 asteroids that yield 261 impact instances - also called virtual impactors (VI) - was calculated. Additionally the method is applied to individual asteroids that were just discovered recently and their risk was determined.

\section{METHOD}

The ARMOR tool works in conjunction with OrbFit [9], a freely accessible software that calculates the ephemeris of potential future impact instances (VI) of observed asteroids. Subsequently, ARMOR projects the spatial impact probability of these impact instances onto the surface of the Earth and this method is explained in more detail in [10]. OrbFit and ARMOR have been used to generate a global impact distribution sample of 261 VIs and the direct impact risk associated with this sample has been calculated in this research.

\section{Risk}

The topic of the asteroid threat is challenging to fathom, let alone communicate, and the reason for this is that the problem describes a low frequency event, which makes it appear insubstantial, but with potentially civilization threatening consequences. Risk expresses the estimated loss of an event which helps to understand the problem more clearly and to make it comparable to similar threats to humans (e.g. other natural disasters). Risk is the product of the probability that an event occurs, exposure the value that is at stake (or exposed) -, and vulnerability the portion of the exposure that is affected. In mathematical terms, this relation can be stated specifically for this application as:

$$
R=P \times \psi \times V(S)
$$

where $R$ is risk to the asteroid threat, $P$ is probability of an asteroid impact, $\psi$ is the population (exposure) and $V(S)$ is the vulnerability which is a function of the severity $S$ of the harmful effects generated by an asteroid impact.

Unsheltered Population - Population is assumed to be the value at stake in this research and these data are available in the form of a global population map for the year 2015 [11] with a grid resolution of $4.6 \times 4.6 \mathrm{~km}^{2}$ (shown in [12]). For subsequent vulnerability analysis, it was necessary to define the average percentage of global population that is unsheltered. Unsheltered population was defined as any population that is outside of buildings and this is relevant to how impact effects may interact with that population.

The literature provides some data about the average time that people spend outdoors but the used data sets are limited to populations that share similar work patterns with the so called "western world". [13] finds that the average American spends $13 \% \approx 3.12$ hours per day outside buildings and the meta study [14] reports that people belonging to western nations spend an average of 1.99 hours per day outdoors which does not include time spent in vehicles. Vehicles offer negligible shelter against thermal radiation as well as shock waves and the time spent in vehicles will be counted towards unsheltered time. Commuting time will be used as a proxy for time in vehicles. The Labour Force [15] reports that the average commuting time in the UK in 2012 was 54.6 minutes. Similarly, the U.S. Census American Community Survey [16] indicates that the average round-trip commuting time in the United States is 50.8 minutes. Adding commuting time as well as the outside 1.99 hours from the meta study provides the time spent outdoors as supported by the meta study and this time is about 2.87 hours or about $12 \%$ of each day. Together, the findings indicate that the average westerner is unsheltered for about $13 \%$ of each day.

The population that this work pattern was applied to is about 2.5 billion people (European Union, USA, Canada, Australia, New Zealand, Japan and parts of: Russia, China, India, Brazil, Argentina, Arab countries), while the global population is about 7.3 billion people. The data reported above does not account for non-western populations. Given the lower industrialisation standard in non-western countries, it is assumed that non-western populations spend twice as long outside as westerners (26\%). With this assumption the western and non-western populations could be connected and the weighted average 
time that the global population spends outdoors was computed to $22 \%$ per day after:

$$
\frac{0.13 \times 2.5+0.26 \times(7.3-2.5)}{7.3}=0.22
$$

For further analysis, it was assumed that $22 \%$ of the global population is unsheltered at any given time.

\section{Impact effect and vulnerability modelling}

Upon colliding with the Earth, an asteroid deposits most of its energy either in the atmosphere, during an airburst, or on the ground by impacting the surface. Whether a ground impact or airburst occurs depends on the entry conditions of the asteroid: impact angle, impact speed, size of the asteroid, and material. In this analysis, impact angle and speed are provided by ARMOR's orbit dynamic impact simulation. Furthermore, size values are published by ESA and NASA on their NEO webpages and the sizes were estimated based on the asteroid's brightness. Finally, the asteroid body was assumed to be similar to ordinary chondrites with a density of $3100 \mathrm{~kg} / \mathrm{m}^{3}$ corresponding to an estimated yield strength of 381315 Pascal (Pa) [17]. Ordinary Chondrites account for about $90 \%$ of all known meteorites [18].

The process that was used to determine if an asteroid experiences an airburst or ground impact is visualized in Figure 1. This process employs analytical models in order to calculate physical processes that occur during atmospheric passage (i.g. break-up altitude, airburst altitude, impact velocity, etc.) as well as the severity of subsequent impact effects. Once the asteroid airbursts or impacts the ground, its energy is released in a variety of impact effects and in this analysis, six impact effects are modelled: High winds, overpressure, thermal radiation, cratering, seismic shaking and ejecta blanket deposition. The first three of these may occur in both, airburst or ground impact, while the latter three only occur in a ground impact.

Upon airburst or impact, the asteroid's kinetic energy is released in the form of impact effects and these impact effects are strongest at the impact site. Starting from the impact site, the effects propagate outwards and attenuate with greater distance. The strength of an effect is called severity and the more severe an effect is, the more likely it is that population is harmed, or, in other words, the vulnerability of the population increases with higher severity.

The following sections describe all six impact effects and their effects on the population. It should be noted that the effect models along with atmospheric passage models are described in greater detail in [17]. However vulnerability models were not readily available, given the particular dependencies required, in this work and most vulnerability models presented here are the result of a combinatory literature review along with evidence based assumptions. A notable resource for vulnerability research is [19] and other sources are indicated where applicable. 


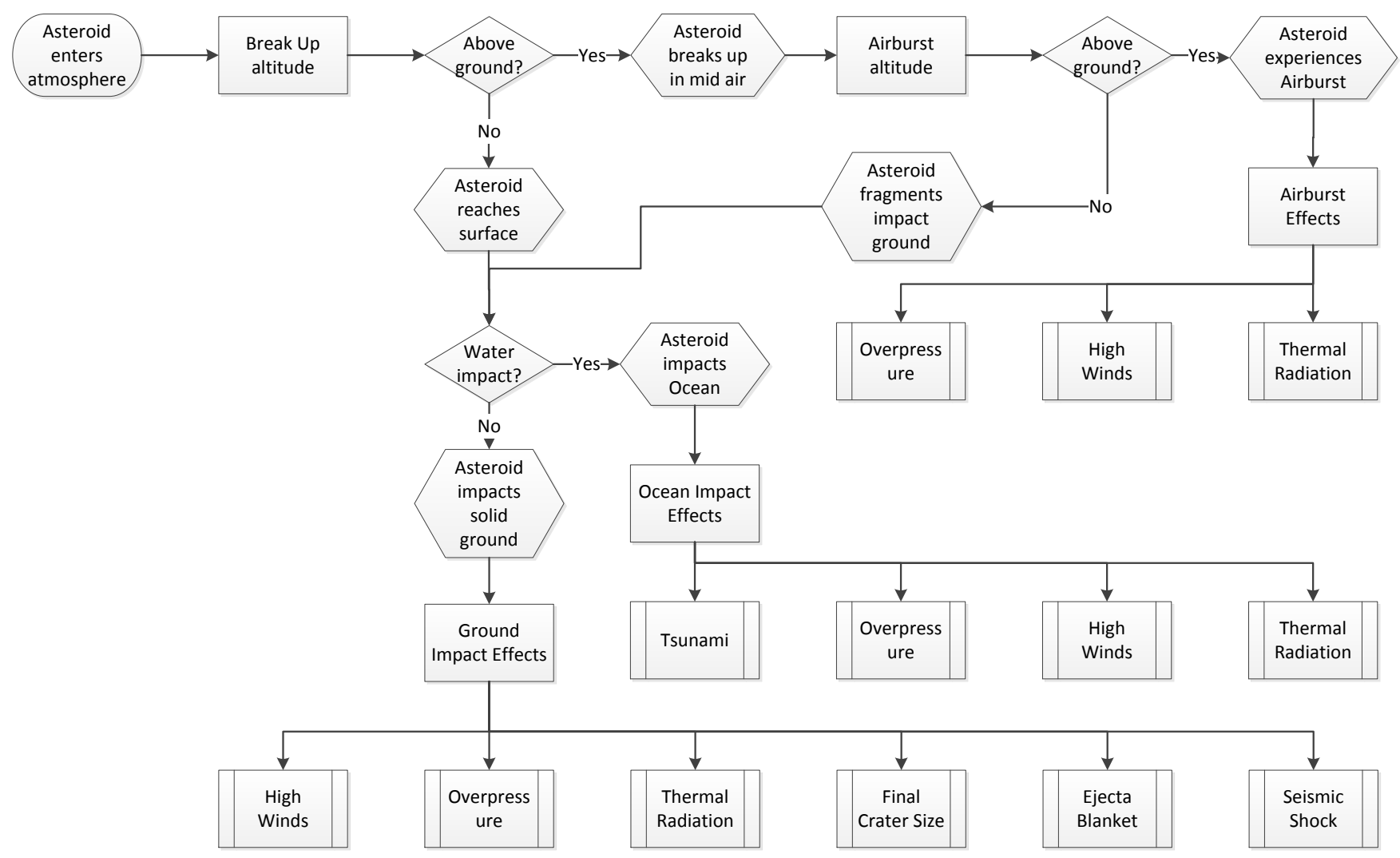

Figure 1: Impact effect flow diagram that shows how an airburst or ground impact is determined and what the corresponding impact effects are. Note that tsunamis are not part of this analysis.

High Winds and Overpressure - The asteroid deposits its energy in an explosion like event that produces an aerodynamic shockwave resulting in a tornado like wind gust and overpressure peak. In accordance with [17], overpressure in a ground impact is calculated as:

$$
p_{D}=\frac{p_{x} D_{x} E_{k t}^{1 / 3}}{4}\left(1+3\left[\frac{D_{x} E_{k t}^{1 / 3}}{D}\right]^{1.3}\right)
$$

where $p_{D}$ is pressure in $\mathrm{Pa}$ at distance $D$ from the impact point in meters, $p_{x}=75000 \mathrm{~Pa}$ and $D_{x}=290 \mathrm{~m}$ are scaling parameters and $E_{k t}$ is the asteroid's kinetic energy at the time of energy deposition in equivalent kilo tons of Trinitrotoluene (kt TNT). In an airburst event, the overpressure shockwave reflects off the surface of the Earth. Directly below the airburst point, a simple shockwave arrives at the surface and overpressure [Pa] is:

$$
p_{D}=p_{0} e^{-\beta D\left(E_{k t}^{-1 / 3}\right)}
$$

where

$$
p_{0}=3.14 \times 10^{11} z_{b 1}^{-2.6}
$$

$$
\beta=34.87 z_{b 1}^{-1.73}
$$

The calculation of energy scaled airburst altitude $z_{b 1}$ is described in [17] as scaling the result of equation 18 using equation 57 of that reference. The pressure shockwave is reflected from the surface and at sufficient distance from the airburst the original and reflected shockwave overlay and interact constructively. In fact, this condition is already described in equation (3) and the switching distance $D_{m 1}$ in meters between equations 4 and (3) is:

$$
D_{m 1}=\frac{550 z_{b 1}}{1.2\left(550-z_{b 1}\right)}
$$

High winds realized in tornado-like wind gusts are a result of the overpressure shockwave and the wind speed $v_{\text {wind }}$ in meters per second after [19] is:

$$
v_{\text {wind }}=\frac{5 p_{D}}{7 p_{a}} \frac{c_{0}}{\left(1+{ }^{6 p_{D} / 7 p_{a}}\right)^{0.5}}
$$

where $p_{a}$ is the ambient pressure and $c_{0}$ is the speed of sound.

The overpressure $p_{D}$ and wind speed $v_{\text {wind }}$ describe the severity of these two impact effects. Effect severity was 
used to determine the vulnerability of the populations that live within the area that is affected by an impact.

Overpressure injures humans by creating a harmful pressure differential between the organ internal pressure (lungs) and ambient pressure. The shockwave rapidly increases ambient pressure leaving the body internals insufficient time to adjust and the resulting pressure differential can rapture tissue.

Vulnerability models are usually represented by sigmoid functions of the form:

$$
V_{\text {effect }}^{\text {case }}(S)=a \frac{1}{1+e^{b(S+c)}}
$$

where $V_{\text {effect }}$ is the vulnerability to a given impact effect, $S$ is the severity of the effect, and $a, b$ and $c$ are constants that are determined in the following sections.

For overpressure vulnerability, three sigmoid functions were fitted to experimental data presented in [19] (Table 12.38). In addition to an expected vulnerability model $V_{p}^{\text {expect }}$, that uses the median values in the table, best $V_{p}^{\text {best }}$ and worst $V_{p}^{\text {worst }}$ case vulnerability functions were derived based on the value ranges provided in the table. The purpose of adding worst and best cases is to gain a sense for the sensitivity of the impact effect models. The resulting overpressure vulnerability $V_{p}$ models are dependent on overpressure (at a given distance) $p_{D}$ and the best fit values for the coefficients $a, b$ and $c$ for the best, expected and worst cases are:

Table 1: Overpressure vulnerability coefficients.

\begin{tabular}{cccc}
\hline Case & $\boldsymbol{a}$ & $\boldsymbol{b}$ & $\boldsymbol{c}$ \\
\hline Expected & 1.0 & $-2.424 \times 10^{-5}$ & $-4.404 \times 10^{5}$ \\
\hline Best & 1.0 & $-1.899 \times 10^{-5}$ & $-5.428 \times 10^{5}$ \\
\hline Worst & 1.0 & $-2.847 \times 10^{-5}$ & $-3.529 \times 10^{5}$ \\
\hline
\end{tabular}

Thus, the vulnerability function to overpressure is:

$$
V_{p}^{\text {case }}\left(p_{D}\right)=\frac{1}{1+e^{b\left(p_{D}+c\right)}}
$$

The vulnerability function is plotted in Figure 2 along with the experimental data points.

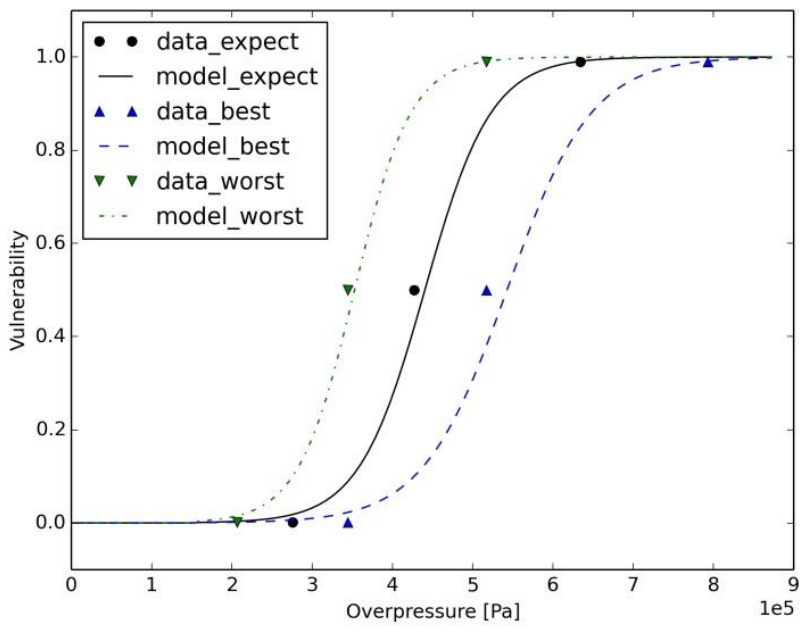

Figure 2: Overpressure vulnerability models with experimental data points.

Strong winds are accompanying the overpressure shockwave and the severity of strong winds is expressed by equation (8). A vulnerability model was derived based on the severity making use of the similarity between strong wind gusts and the criteria in the Enhanced Fujita (EF) scale which is used to classify tornado strength [20]. In the EF scale, tornados are classified based on the damage that they cause during a 3 seconds wind gust and Table 2 provides an overview over EF category, wind speed and expected damage.

Table 2: Enhanced Fujita scale. Categories, wind speeds and damage

\begin{tabular}{lll}
\hline Category & $3 s$ Wind Gust $[\mathrm{m} / \mathrm{s}]$ & Typical Damage \\
\hline EF0 & $29-38$ & Large tree branches broken; \\
& & Trees may be uprooted; Strip \\
& mall roofs begin to uplift. \\
\hline EF1 & $38-49$ & Tree trunks snap; Windows in \\
& & Institutional buildings break; \\
& Facade begins to tear off. \\
\hline EF2 & $49-60$ & Trees debark; \\
& & transmission line towers break; \\
& Family residence buildings \\
& severely damaged and shift off \\
& foundation. \\
\hline EF3 & Metal truss transmission towers \\
& collapse; Outside and most inside \\
& walls of family residence \\
& buildings collapse. \\
\hline EF4 & $74-89$ & Severe damage to institutional \\
& building structures; All family \\
& & residence walls collapse. \\
\hline EF5 & Severe general destruction. \\
\hline
\end{tabular}

EFO - According to the EF scale, EF0 corresponds to wind speeds between $29-38 \mathrm{~m} / \mathrm{s}$. Humans can be harmed in this condition by being thrown against objects or objects being hurled at them. In [19] lethality estimates are provided for 
objects turned missiles which hit the body. According to this source, a $5 \mathrm{~kg}$ object entails a near $100 \%$ rate of fracturing a skull when hitting the head with a velocity exceeding $7 \mathrm{~m} / \mathrm{s}$. On the other hand, lethality may occur when the body is thrown against solid objects with velocities in excess of $6 \mathrm{~m} / \mathrm{s}$. It is conceivable that these events may be produced in a category EF0 tornado and, indeed, category EF0 tornados have been lethal in the past [21] but the casualty rate is low (3 people were killed by EF0 tornados between 1997 and 2005). Here, it was assumed that $1 \%$ of the population that is outdoors is being hit by missiles or thrown against objects and that $10 \%$ of these individuals die. These assumptions provide a vulnerability of 0.00022 for strong winds corresponding to a category EF0 tornado.

EF1 - Housing still provides decent protection against high winds corresponding to EF1tornados and the assumption that only the outdoor population is affected was kept. However, a larger portion of the outside population will be affected; more people will be thrown against solid objects because the strong wind will be able to lift up more people. The wind will also generate more missiles that could hit people. Furthermore, the lethality for each person also increases because the impact speed of the body or the missile will be higher. [19] estimates that $50 \%$ is reached when a body contacts a solid object with a speed of $16.5 \mathrm{~m} / \mathrm{s}$ and $100 \%$ lethality is reached at $42 \mathrm{~m} / \mathrm{s}$. It seems plausible that a body could be accelerated to speeds of 16.5 - $42 \mathrm{~m} / \mathrm{s}$ in an EF1 tornado. However, it can be assumed that some of the outside population finds sufficient shelter. Hence, it was assumed that $20 \%$ of the outside population is affected and that $50 \%$ of those affected die. Vulnerability for winds corresponding to an EF1 tornado is, thus, set to 0.022 .

The assumed increase in vulnerability agrees well to the increase of lethality of recorded tornados between 2000 and 2004 [21]. During that time period 4284 EF0 tornados killed 2 people resulting in a casualty rate of 0.00047 per EF0 tornado. In the same time 1633 EF1 tornados killed 20 people yielding a casualty rate of 0.012 per EF1 tornado which is an increase of two orders of magnitude. Similarly, assumed vulnerability for strong winds also increased by two orders of magnitude.

EF2 - Increasing wind speed renders shelters less effective as houses start to exhibit significant damage. It is assumed that in addition to the outside population, some portion of the housed population is affected yielding a total of $30 \%$ exposed population. A larger portion of the exposed population will be affected by increasing wind speeds and this portion is assumed to be $60 \%$. Lethality for affected population also increases to an assumed $80 \%$ as wind speeds are capable of accelerating bodies beyond the 42 $\mathrm{m} / \mathrm{s}$ body impact speed assumed for $100 \%$ lethality [19] and objects turned missiles have higher damage potential. Consequently, vulnerability is equal to 0.144 .

The increase in vulnerability from EF1 to EF2-like wind speeds of one order of magnitude matches the casualty rate increase from EF1 to EF2 tornados. Between 2000 and 2004, $439 \mathrm{EF} 2$ tornados killed 51 persons yielding a casualty rate of 0.116 per EF2 tornado corresponding to an order of magnitude increase.

EF3 - Tornados of this category destroy most housing shelter leaving basements and well-constructed concrete buildings as viable shelter options. It was assumed that $50 \%$ of the population would be exposed to winds of this strength, irrespective of whether the population is inside or outside. Of those exposed, $80 \%$ would be lethally affected by missiles or by being thrown against fixed structures. The vulnerability thus increases by and order of magnitude to 0.4 . The record shows that 116 persons were killed by 127 EF3 tornados yielding a casualty rate of 0.913 that corresponds to an order of magnitude increase from EF2 to EF3 tornados.

EF4 - Persons who are sheltered in very well constructed concrete buildings will be protected against these winds. It was assumed that $90 \%$ of the population would be exposed and that $95 \%$ would die. Thus, vulnerability is 0.855 .

EF5 - The great majority of structures collapses in these winds offering insignificant protection. Consequently, it is assumed that vulnerability is $95 \%$ for the entire population at $90 \mathrm{~m} / \mathrm{s}$ wind speed and that $100 \%$ die in wind speeds greater than $100 \mathrm{~m} / \mathrm{s}$.

Based on these data, three vulnerability models were derived: One model that describes the expected case $V_{\text {wind }}^{\text {expect }}$ and two for a worst $V_{\text {wind }}^{\text {worst }}$ and best $V_{\text {wind }}^{\text {best }}$ case. The expected case uses the median wind speed for each EF category with the corresponding vulnerability value, while the worst and best case models use the wind speeds of one category lower or higher, respectively. The model function is:

$$
V_{\text {wind }}^{\text {case }}\left(v_{\text {wind }}\right)=\frac{1}{1+e^{b\left(v_{\text {wind }}+c\right)}}
$$


and the corresponding coefficients are:

Table 3: Wind vulnerability coefficients.

\begin{tabular}{cccc}
\hline Case & $\boldsymbol{a}$ & $\boldsymbol{b}$ & $\boldsymbol{c}$ \\
\hline Expected & 1.0 & $-1.343 \times 10^{-1}$ & $-6.944 \times 10^{1}$ \\
\hline Best & 1.0 & $-1.279 \times 10^{-1}$ & $-7.639 \times 10^{1}$ \\
\hline Worst & 1.0 & $-1.429 \times 10^{-1}$ & $-6.246 \times 10^{1}$ \\
\hline
\end{tabular}

Figure 3 shows the vulnerability models plotted over the relevant range of wind gust speeds.

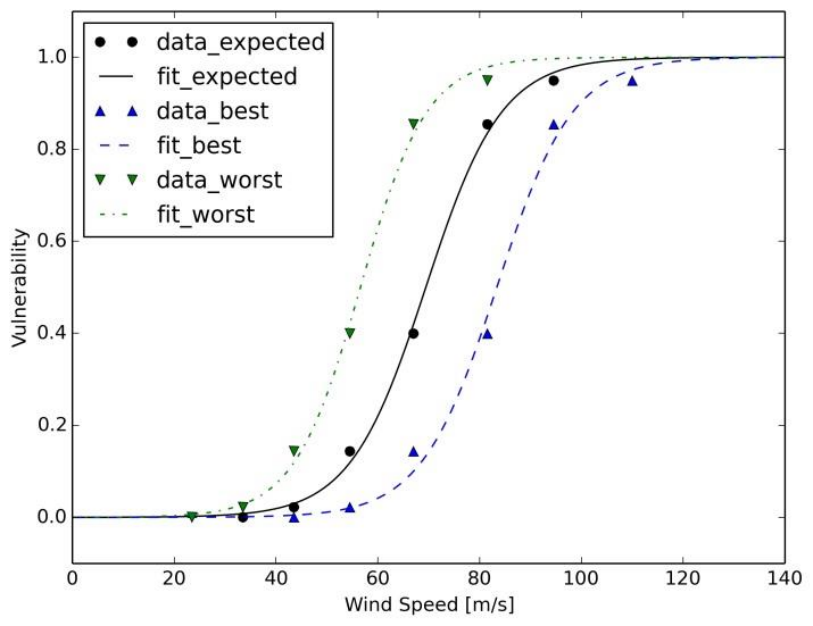

Figure 3: Wind vulnerability models with data points.

Thermal Radiation - Ground impacts as well as airburst produce thermal radiation but the two events require separate modelling as presented in the following.

Ground Impact - If the impacting meteoroid travels in excess of $15 \mathrm{~km} / \mathrm{s}$, enough energy is released to evaporate the asteroid and some of the ground material. This violent event generates a plume with very high pressure $(>100 \mathrm{GPa})$ and temperature $(\approx 10000 \mathrm{~K})$ that rapidly expands. This is called the fireball. As a result of the high temperature, the gas is ionized and appears opaque to thermal radiation due to the plasmas radiation absorption characteristics. Consequently, the plume expands adiabatically and only starts to radiate outwards when the plasma cools to the transparency temperature $T_{*}$ [22]. Reference [17] reports an empirical relationship for the fireball radius $R_{f}$ when it reaches transparency temperature as a function of impact energy $E$ :

$$
R_{f}=0.002 E^{1 / 3}
$$

Only a fraction of the kinetic energy released during impact is transformed into thermal radiation [23]. This fraction is called luminous efficiency $\eta_{\text {lum }}$ and [24] determined that it is on the order of $10^{-4}$ to $10^{-2}$. The received thermal energy per area unit (assuming a hemispheric dissipation of heat radiation) is given by [17] as:

$$
\phi=f \frac{\eta_{l u m} E}{2 \pi D^{2}}
$$

Where, $f$ is the fraction of the fireball that is visible over the horizon at distance $D$ which is also a function of $R_{f}$ and the corresponding geometric relationship is given in [17] (Equation 36).

Airburst - Besides the air blast, some of the kinetic energy carried by the meteoroid that is released during airburst dissipates as thermal radiation. [25] investigated the radiation emitted by meteors and the following airburst thermal radiation model was derived here based on this research. Equation (11) of the reference provides an expression for thermal energy flux density based on airburst intensity:

$$
\phi=q_{h}\left[\frac{L_{0}}{D_{\text {los }}}\right]^{2} 5
$$

where $\phi$ is the energy flux density in $\left[\mathrm{W} / \mathrm{m}^{2}\right]$ at the target distance, $q_{h}$ (the reference uses $q_{\infty}$ ) is the energy flux density of the meteoroid at a given altitude, $L_{0}$ is the asteroid diameter and $D_{l o s}$ is the line of sight distance from the airburst to the target. Table 1 of the reference provides values for $q_{h}$ as a function speed for the two altitudes of $25 \mathrm{~km}$ and $40 \mathrm{~km}$. Here, an interpolation function was built that produces $q_{h}$ values for any given speed, altitude pair based on table 1 in the reference. To this end, a six degree polynomial was least square fitted to the data describing $q_{h=25}$ at $25 \mathrm{~km}$ altitude as a function of meteoroid speed $v$ :

$$
\begin{gathered}
q_{h=25}=\left(-4 \times 10^{-16} v^{6}\right) \\
+\left(7 \times 10^{-11} v^{5}\right)-\left(5 \times 10^{-6} v^{4}\right) \\
+\left(0.176 v^{3}\right)-\left(3160.6 v^{2}\right) \\
+\left(3 \times 10^{7} v\right)-1 \times 10^{11}
\end{gathered}
$$

The polynomial has a correlation coefficient of 0.9868 with the data. Only three data points were available for the data for $q_{h=40}$. However, the data fits perfectly on a line described by:

$$
q_{h=40}=700000 v-1 \times 10^{10}
$$

Finally, a linear interpolation scheme estimates $q_{h}$ for any given airburst altitude $z_{b}$ based on the calculated values for $q_{h=25}$ and $q_{h=40}$. 


$$
q_{h=z_{b}}=\frac{q_{25}-q_{40}}{15000}\left(40000-z_{b}\right)+q_{40}
$$

The distance $D_{\text {los }}$ was estimated using Pythagoras' relationship with airburst altitude $z_{b}$ and ground distance $D$ as parameters:

$$
D_{\text {los }}=\sqrt{z_{b}^{2}+D^{2}}
$$

With these relations, Equation (14) can be solved and a thermal energy flux density may be obtained for any airburst event.

Note that the unit of Equation (14) is $\left[\mathrm{W} / \mathrm{m}^{2}\right]$ and that for subsequent analysis the thermal radiation energy density $\left[\mathrm{J} / \mathrm{m}^{2}\right]$ was needed. Based on visual observations of the Chelyabinsk [4] and other meteors [26] it was determined that a break-up occurs within a time span on the order of one second. Therefore, one second was assumed as the default break-up duration for airbursts and the unit $\left[\mathrm{W} / \mathrm{m}^{2}\right]$ is equivalent to the energy density $\left[\mathrm{J} / \mathrm{m}^{2}\right]$ when integrated for this timespan because energy is the integral of energy flux $[\mathrm{W}]=[\mathrm{J} / \mathrm{s}]$ over time [s]. This relation is expressed by the following example equation assuming that energy flux is constant over time:

$$
1 \mathrm{~J}=1 \mathrm{~W} \times 1 \mathrm{~s}=1 \mathrm{~J} / \mathrm{s} \times 1 \mathrm{~s}
$$

Vulnerability Model - Thermal radiation is emitted from airbursts and ground impacts. Surfaces that are incident to the radiation heat up and can be burned or ignited. The consequences of thermal radiation energy exposure on the human body as a consequence of nuclear detonations were investigated in [19] and serves as basis for the thermal radiation vulnerability model. It should be noted that the spectral intensities in the burn relevant portion of asteroid and nuclear explosion generated radiation spectra will differ from each other. This could lead to non-identical efficiencies in translating radiation energy into burn injury. However, given the sparse evidence basis of asteroid explosions and few literature sources, the approach presented here represents a best effort to treat asteroid caused radiation vulnerability. The burn probability as a function of radiant exposure and explosion yield is given in Figure 12.65 of the reference. While the dependency of burn probability to radiant exposure $\left[\mathrm{J} / \mathrm{m}^{2}\right]$ is obvious, its dependency on explosion yield should be explained.

The dependency on explosion yield is rooted in the observation that the process of small yield explosions takes less time to unfold than large yield explosions resulting in different energy flux rates. For smaller explosions, a given amount of radiant energy is delivered in a shorter time compared to a larger explosion and, thus, the radiation intensity differs with explosion yield. Higher radiation intensity causes injuries more readily than low intensity radiation even though the same cumulative energy might be delivered in both cases. The reason for this behaviour is that the heated surface has more time to dissipate the incident radiation energy in a low intensity radiation case. Unlike nuclear explosive devices, meteoroids are not optimized for explosion and it is thus assumed that their explosion signature is more comparable to that of a large nuclear device because the explosion process takes relatively long. The data used to build the vulnerability model correspond to the results produced by a 1Mton TNT equivalent yield nuclear device as shown in Figure 12.65 in [19].

The burn severity distribution is a function of radiant exposure and the data in the reference forms the basis for Figure 4 that shows which burn degree can be expected when exposed to a certain radiant energy.

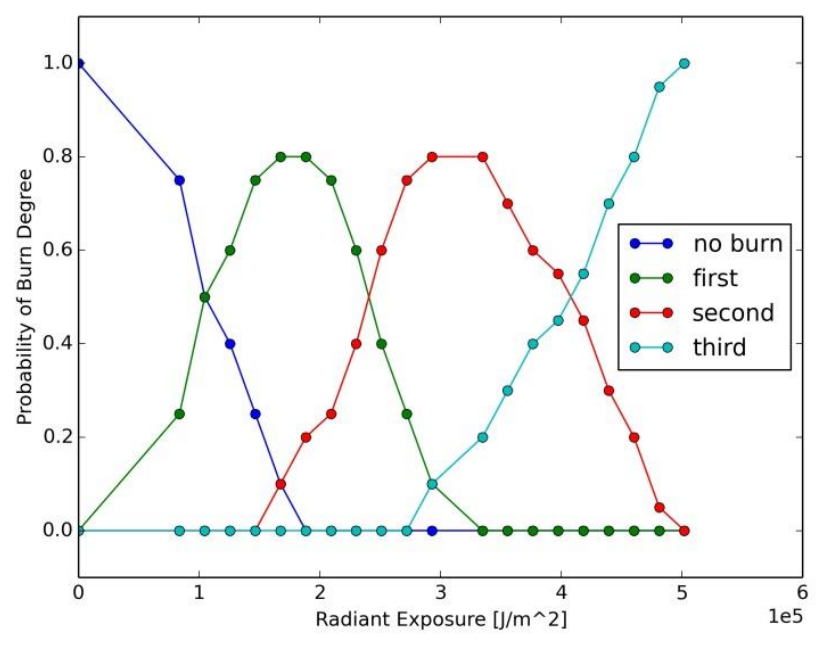

Figure 4: Burn degree distribution as a function of radiation intensity based on data in [19] assuming the explosion signature of a 1Mton TNT yield nuclear device.

Aside from burn degree, the total body surface area (TBSA) of a human that is burned determines the expected mortality. In [27], statistical analysis of 143199 burn victims in the United States have been analysed for their mortality rate based on burned TBSA. The reported numbers apply to persons who have been treated in medical facilities after the burn injury. This means that the burn injury itself could be treated adequately but also that 
possible subsequent medical complications (pneumonia, infection) that are directly linked to the burn injury could be addressed. Here, it shall be assumed that mortality rates are twice as high because proper and timely treatment of burn injuries is unlikely in the event of an asteroid impact that will potentially affect a large region and its medical infrastructure. Figure 5 visualizes the data in Table 9 of [27] and shows the mortality rate as a function of burnt TBSA for treated and untreated victims.

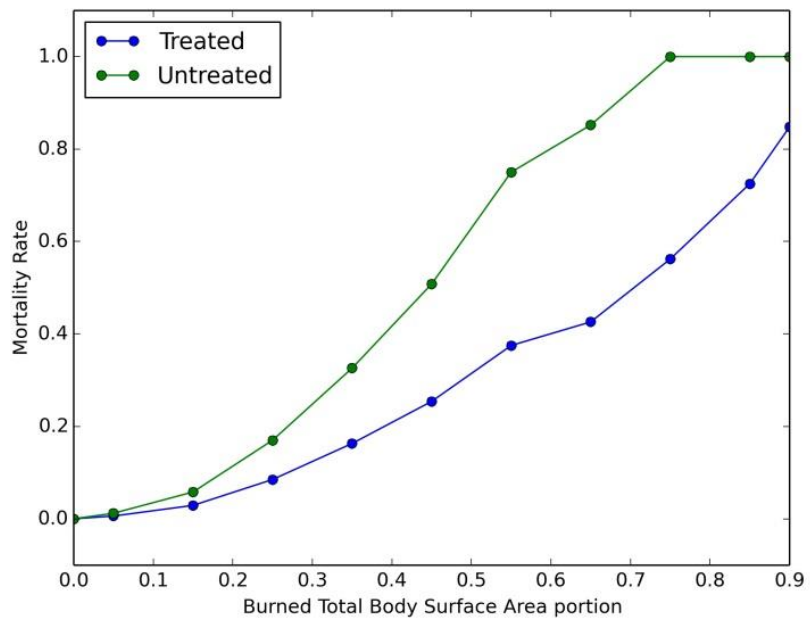

Figure 5: Mortality rate for treated and untreated burn victims as a function of burnt TBSA. Data from [27].

To relate radiant exposure to TBSA and, thus, to mortality rate, a scaling law is introduced that approximates TBSA based on the burn degree distribution as a function of radiant exposure. In general, every part of the body that is exposed to light from the meteoroid explosion will be burned, but the severity of the burn differs. A superficial first degree burn, which is comparable to a bad sun burn, is less life threatening than a third degree burn that penetrates through all skin layers. To account for this distinction, a scaling law was introduced the yields TBSA as a function of burn degree distribution. The scaling law is the weighted sum (first degree has weight one, second degree has weight two and third degree has weight three) of the burn distribution as a function of radiant exposure.

$$
\begin{aligned}
\operatorname{TBSA}_{\text {weighted }}(\phi) & =\frac{1}{9}\left[1 \times \operatorname{burn}_{1^{\circ}}(\phi)+2\right. \\
& \times \operatorname{burn}_{2^{\circ}}(\phi)+3 \\
& \left.\times \operatorname{burn}_{3^{\circ}}(\phi)\right]
\end{aligned}
$$

Furthermore, the scaling law respects the observation that the thermal radiation from an asteroid impact arrives from only one direction. This situation allows to establish that only half of a human, or a maximum of $50 \%$ TBSA, can be injured from thermal radiation. Moreover, clothing (as long as it does not burn itself) provides protection against a short lived energy burst of thermal radiation and it is therefore assumed that only one third of TBSA can be burned for people standing outside. Figure 6 visualizes the resulting TBSA curve as a function of radiant exposure.

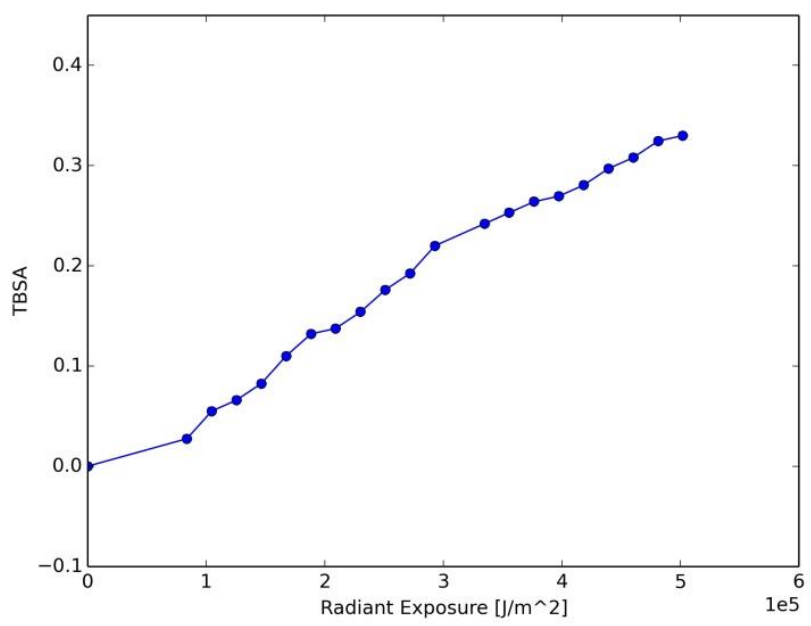

Figure 6: Visualization of TBSA-burn degree scaling law (Equation 20). The maximum TBSA is scaled to one third as clothing offers protection and radiation comes from one direction.

Combining the data from [19] about radiant exposure and the resulting burn severity with the scaling law to relate burn severity with TBSA and, finally, with the data from [27] about mortality rate based on TBSA, mortality rate can be expressed as a function of radiant exposure. Figure 7 shows the relationship. This data is based recorded occurrences and the corresponding radiant exposure range is limited to these records. An asteroid impact can produce higher radiant energies and the mortality rate, thus, has to be expanded to higher values of radiant exposure. 


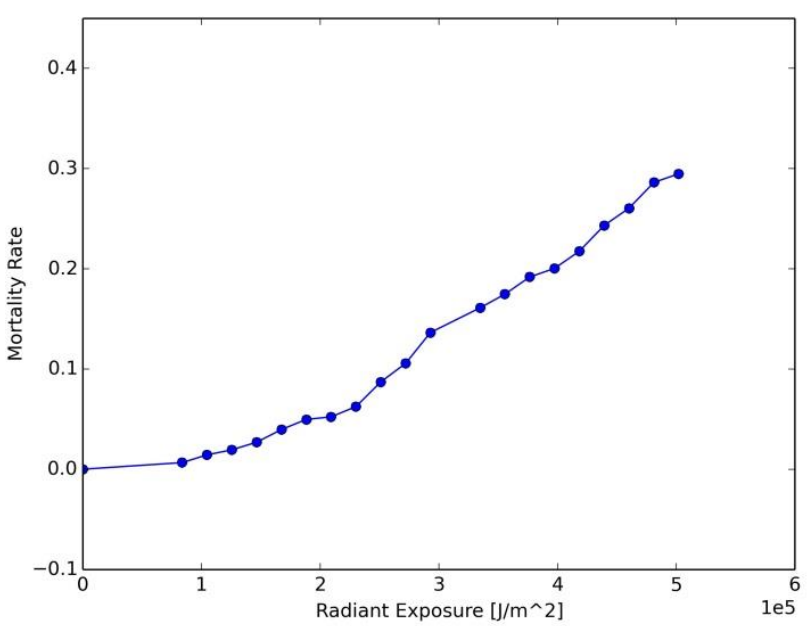

Figure 7: Mortality rate as a function of radiant exposure.

Clothing provides limited protection to thermal radiation because it can absorb thermal energy up to the point when it itself ignites and it was therefore assumed that only one third of TBSA can be burned before clothing ignites. [19] reports that cotton and denim clothing ignites at about $836800 \mathrm{~J} / \mathrm{m}^{2}$. Beyond this energy level clothing does not offer protection and it was assumed that $50 \%$ of TBSA can be burned resulting in a mortality rate of $62 \%$ [27]. Furthermore, at energy densities of $1255200 \mathrm{~J} / \mathrm{m}^{2}$, [19] reports that sand explodes (popcorning), aluminium aircraft skin blisters and roll roofing material ignites. These conditions appear lethal to humans and a mortality rate of one is assumed for a population exposed to this energy level.

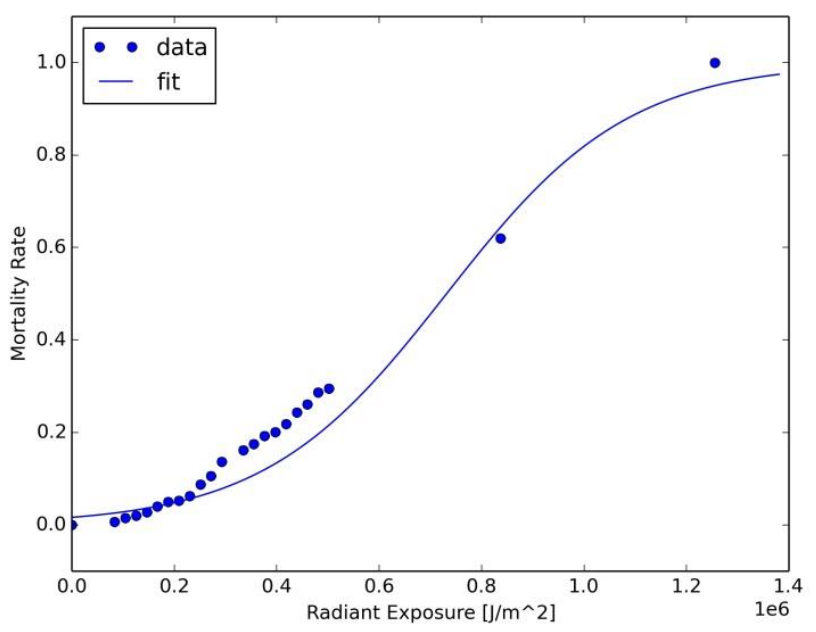

Figure 8: Mortality rate as a function of the full, applicable radiant exposure range.

Figure 8 presents the full range of thermal radiation mortality rate and shows the corresponding data points.
Additionally, a sigmoid function has been least square fitted to the data and the corresponding mathematical description is:

$$
\begin{gathered}
\text { Mortality Rate }(\phi)= \\
=\frac{1}{1+\mathrm{e}^{-0.00000562327(\phi-731641.664)}}
\end{gathered}
$$

The mortality numbers derived above apply to exposed population that is outside of sheltering buildings. For people inside of buildings the mortality rate will be moderated through the protective effect of walls. However, windows do not offer protection against thermal radiation and it is assumed that one third of the inside population (25\% of global population) is exposed through windows even though they are inside a building. The expected case is that $22 \%$ are outside and $25 \%$ are exposed behind windows (totalling 47\%) while the remaining $53 \%$ of the global population are unaffected by thermal radiation. The mathematical expression for thermal radiation vulnerability is, thus:

$$
V_{\phi}^{\text {case }}(\phi)=a \frac{1}{1+e^{b(\phi+c)}}
$$

For the expected case, a maximum of $47 \%$ of the population is exposed. Additionally, in the worst case scenario it was assumed that the entire population is outdoors (exposed) while in the best case scenario the entire population is sheltered and the corresponding coefficients are:

Table 4: Thermal radiation vulnerability coefficients.

\begin{tabular}{cccc}
\hline Case & $\boldsymbol{a}$ & $\boldsymbol{b}$ & $\boldsymbol{c}$ \\
\hline Expected & 0.47 & $-5.623 \times 10^{-6}$ & $-7.316 \times 10^{5}$ \\
\hline Best & 0.25 & $-5.623 \times 10^{-6}$ & $-7.316 \times 10^{5}$ \\
\hline Worst & 1.0 & $-5.623 \times 10^{-6}$ & $-7.316 \times 10^{5}$ \\
\hline
\end{tabular}

Figure 9 visualizes these vulnerability models. 


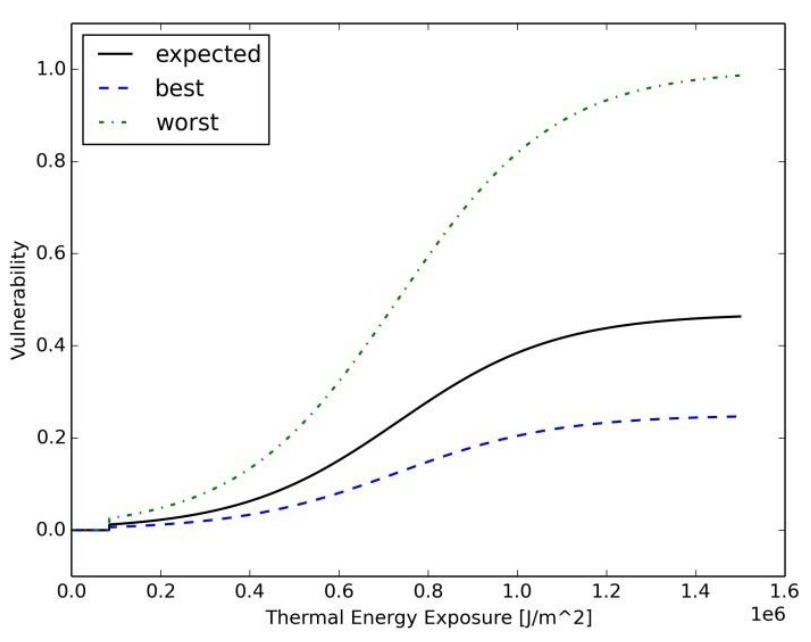

Figure 9: Thermal radiation vulnerability models.

Cratering - When a meteoroid impacts the ground, an impact crater forms. The cratering process is complex in itself and occurs in several steps. In a first step, a transient crater is formed which is the dynamical response to the impacting meteoroid. It is useful to calculate the transient crater because the final crater shape depends on the intermediate step of the transient crater. In fact, the energy delivered by the asteroid is so big and the speed of the mechanical interaction between asteroid and ground is so fast that the target material (water or ground) react like a fluid and thus can be described with the same formalism. A transient crater is generally an unstable structure and is similar to the crown-like shape that forms in a water surface immediately after a droplet falls into it. The "crown ring" surrounds the impact point that forms a bowl shaped depression and represents the crater bottom. A transient crater is not self-supporting and collapses under the influence of gravity to form the final crater shape. The transient crater diameter $D_{t c}$ is given in [17] with:

$$
D_{t c}=1.161\left(\frac{\rho_{i}}{\rho_{t}}\right)^{1 / 3} L_{0}^{0.78} v_{i}^{0.44} g_{0}^{-0.22} \sin ^{1 / 3} \gamma
$$

where $\rho_{i}$ is the impactor density, $\rho_{t}$ is target (ground) density (assumed to be $2500 \mathrm{~kg} / \mathrm{m}^{3}$ ), $v_{i}$ is impactor speed, $g_{0}=9.80665 \mathrm{~m} / \mathrm{s}^{2}$ is Earth standard gravity and $\gamma$ is the impactor angle (an impact velocity vector normal to the ground corresponds to $\gamma=90^{\circ}$ ).

With the collapse of the transient crater, the final crater forms. Equation 27 in [17] provides a mathematical description of final crater diameter. In a sensitivity analysis, it was determined that the final crater diameter
$D_{f r}$ is typically $25 \%$ bigger than the transient crater diameter. The sensitivity analysis considered impact speed, impact angle, asteroid density and target density. This estimate is found to be accurate to within $5 \%$ for asteroids in the diameter regime of $0-500 \mathrm{~m}$. The same conclusion was reached in [17] and the final crater diameter is:

$$
D_{f r}=1.25 D_{t c}
$$

Determining the vulnerability of the population due to crater formation was straight forward. People who were located within the final crater zone at the time of impact had no chance of survival and, thus, vulnerability was unity in this area. On the other hand, people outside the final crater zone were not affected by cratering. In this research, world grid data was employed that exhibit a cell resolution of about $4.6 \times 4.6 \mathrm{~km}^{2}$. Cratering vulnerability in a given grid cell was determined by calculating the fraction of the crater area that covers this specific grid cell with respect to the grid cell area. Note that the impact point grid cell might be covered completely by the crater but that cells that are located on the rim of the crater are only partially covered and this algorithm accounts for such situations. To this end, the final crater area was assumed to be circular:

$$
A_{f r}=\pi\left(\frac{D_{f r}}{2}\right)^{2}
$$

Seismic Shaking - The seismic shock is expressed in terms of the Gutenberg-Richter scale magnitude. It is assumed that a fraction of $10-4$ of the impacting kinetic energy is transformed into seismic shaking [28]. The Gutenberg-Richter magnitude energy relation provided the magnitude of the expected shock as:

$$
M=0.67 \log _{10} E-5.87
$$

Where $E$ is the impacting kinetic energy in Joules, and $M$ is the magnitude on the Richter scale. With increasing distance from the impact site, the force of the shocks decreases and [17] present an empirical law that describes the effective magnitude $\mathrm{M}$ eff at a distance $D$ from the impact site:

$$
\begin{gathered}
M_{\text {eff }}= \\
\left\{\begin{array}{c|c}
M-2.38 \times 10^{-5} D & D<60 \mathrm{~km} \\
M-4.8 \times 10^{-6} D-1.1644 & 60 \leq D<700 \mathrm{~km} \\
M-1.66 \log _{10} \Delta-6.399 & 700 \mathrm{~km} \leq D
\end{array}\right.
\end{gathered}
$$


The effective magnitude can be related to the expected destruction at the given distance from the impact point and determines vulnerability.

A literature review has been conducted to find suitable data to support a seismic vulnerability model. Specifically, data was needed to relate seismic shaking magnitude at a given location to the mortality rate at this location. However, typical earthquake records only report peak intensity and total losses and this data is too convoluted for usage here because it depends on population density and affected area in the location of the earthquake which are typically not reported in the respective publications [29]. The data reports fatalities that occur in an area that encompasses the entire earthquake region and relates this casualty figure to the peak intensity shaking. However, not all fatalities occur at the location of peak shaking intensity (the epicentre) and some casualties are found at a distance away from the epicentre. Thus, it would be wrong to use this data because it attributes the casualties of the entire earthquake region to the peak shaking intensity and would produce an overestimation for a given seismic intensity.

What was needed is a function that provides the mortality rate with respect to local shaking magnitude because mortality varies with distance from the epicentre. In other words, the same earthquake produces a high mortality rate close to the epicentre where seismic shaking is severe and a lower mortality rate at a distance from the epicentre because seismic shaking attenuates with greater distance. In [30], mortality rates are provided as logistic functions with respect to seismic intensity based on earthquake records in China and these functions were validated against four severe earthquake events. It should be noted that the reported mortality rates are equivalent to the vulnerability rates that are of interest here because the mortality rates describe the observed result of how many casualties occurred for a given seismic shaking intensity. The vulnerability logistic function that best fit the validation data (mean estimation error of 12\%) with seismic intensity (Modified Mercalli Intensity [31]) as free parameter is:

$$
V_{i_{\text {seis }}}=\frac{1}{0.01+2.691 \times 10^{6} \times 0.170^{i_{\text {seis }}}}
$$

In table 2 of [17], the necessary data to translate Modified Mercalli Intensity into Richter scale magnitude values is provided. Here, a linear function was least square fit to the data $\left(R^{2}=0.9887\right)$ and it is:

$$
i_{\text {seis }}=1.4199 M_{\text {eff }}-1.3787
$$

Equations (28) and (29) can be combined into a new sigmoid function that yields expected vulnerability with effective shaking expressed in Richter scale magnitude as free parameter:

$$
V_{\text {seis }}^{\text {case }}=\frac{1}{1+e^{-2.51607678(M e f f-8.68559246)}}
$$

Furthermore, Figure 4 as well as Table 5 of [30] supplies data about the variability in vulnerability data. Based on these additional information the curves for best and worst case vulnerability to seismic shaking could be established, and the corresponding coefficients are:

Table 5: Seismic shaking vulnerability coefficients.

\begin{tabular}{cccc}
\hline Case & $\boldsymbol{a}$ & $\boldsymbol{b}$ & $\boldsymbol{c}$ \\
\hline Expected & 1.0 & $-2.516 \times 10^{0}$ & $-8.686 \times 10^{0}$ \\
\hline Best & 1.0 & $-2.508 \times 10^{0}$ & $-9.590 \times 10^{0}$ \\
\hline Worst & 1.0 & $-3.797 \times 10^{0}$ & $-7.600 \times 10^{0}$ \\
\hline
\end{tabular}

Figure 10 shows the seismic vulnerability functions over an expected range of seismic shaking magnitudes.

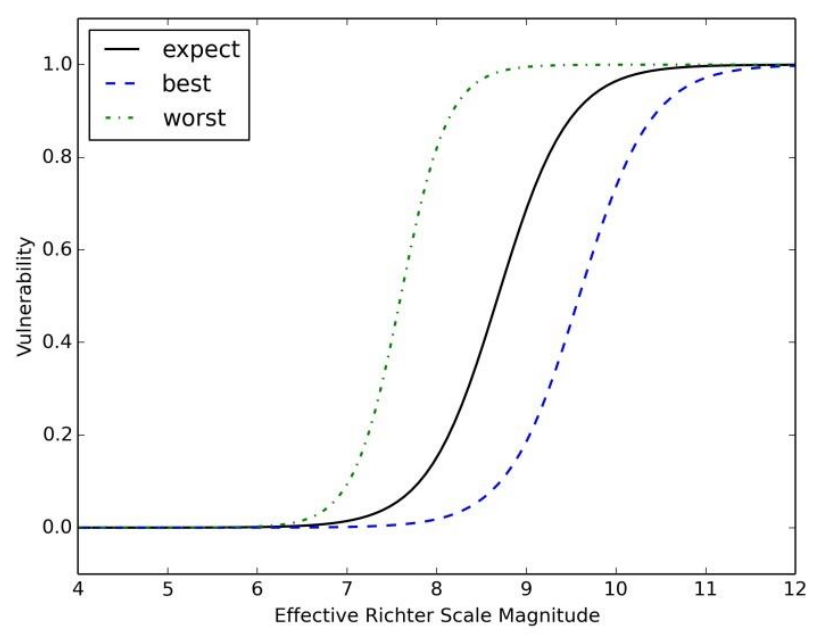

Figure 10: Seismic shaking vulnerability models as a function of effective Richter scale magnitude.

Ejecta Blanket Deposition - In addition to plastically deforming and partially melting the impact site, the asteroid impact also ejects ground material outwards from its impact site and the removed material is called ejecta. Here, the ejecta blanked deposition was modelled which 
can lead to delayed damage such as building collapse due to the accumulating ejecta load on structures. [17] derive an analytical expression for ejecta blanket thickness $t_{e}$ as a function of transient crater size $D_{t c}$ and distance from impact site $D$ :

$$
t_{e}=\frac{D_{t c}^{4}}{112 D^{3}}
$$

Ejecta deposition is a hazard because it can lead to the collapse of buildings if the weight load of the settling ejecta blanket becomes big enough. The vulnerability model used in this work follows closely the method described in [32] and a mean ejecta material density of $\rho_{e}=1600 \mathrm{~kg} / \mathrm{m}^{3}$ is assumed. Given the ejecta density $\rho_{e}$, ejecta blanket thickness $t_{e}$ and the standard gravitational acceleration $g_{0}$, the load of the ejecta blanket is:

$$
p_{e}=t_{e} \rho_{e} g_{0}
$$

In [33] it is estimated that $20 \%$ of the occupants in a house would be trapped in the event of a collapse and half of those would be fatalities. Keeping with previous assumptions that $22 \%$ of the population would be outside at any given time, the remaining $78 \%$ are located indoors. Taking these factors together, the maximum vulnerability of the population in the event of a roof collapse is $0.78 \times 0.2 \times 0.5=0.078$. However, to realize this vulnerability the roof of a building has to collapse in the first place. The likelihood of roof collapse can be modelled as a function of ejecta load as well as building strength and the corresponding models have been derived in [33] The resulting vulnerability model for the expected case that assumes medium strength housing is:

$$
V_{e}^{\text {expect }}=0.078 \times\left[1+e^{-1.37\left(p_{e}-3.14\right)}\right]^{-4.6}
$$

In the best and worst case models, strong and weak building strengths were assumed, respectively. The corresponding vulnerability models are:

$$
\begin{aligned}
V_{e}^{\text {best }} & =0.078 \times\left[1+e^{-1.00\left(p_{e}-5.84\right)}\right]^{-2.58} \\
V_{e}^{\text {worst }} & =0.078 \times\left[1+e^{-4.32\left(p_{e}-1.61\right)}\right]^{-4.13}
\end{aligned}
$$

Figure 11 visualizes the vulnerability models as a function of ejecta blanket thickness.

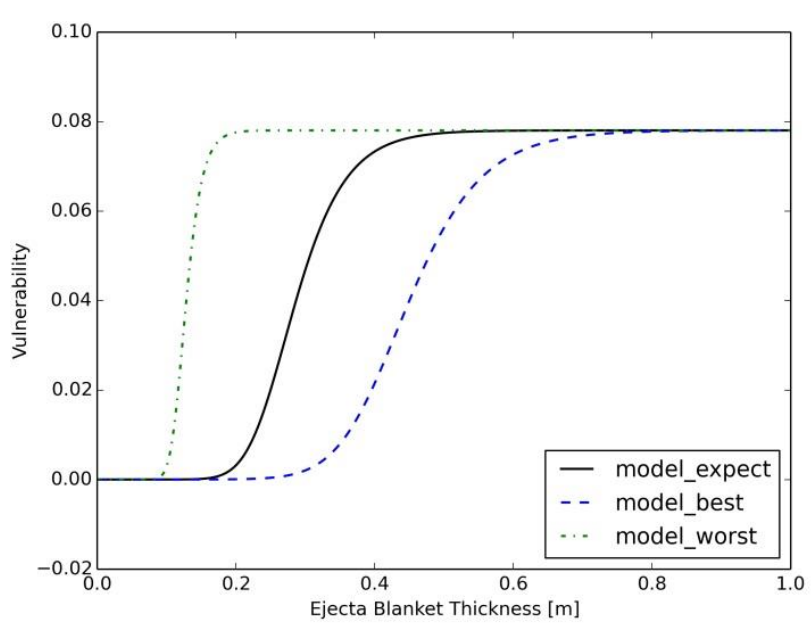

Figure 11: Ejecta blanket thickness vulnerability models.

\section{RESULTS}

The sample of 261 VIs was used to calculate the global risk of this sample and Figure 12 visualizes the risk distribution while the corresponding 261 impact corridors are visualized in [34]:

Total risk in the expected case is 13.594719 casualties for this sample considering potential impacts until the year 2100. In contrast, the best case yields a global risk of 10.291979, while the worst case scenario yields 18.191418 casualties. Note that these results do not include the modelling of tsunamis and their effects which are expected to raise risk estimates.

Two aspects are notable in Figure 12: First, the risk distribution is similar to the global population distribution and, second, some high impact probability and big asteroids yield high risk and produce impact corridor shaped local risk maxima.

$2015 R N 35$ - In a more direct application, the risk of asteroid, "2015 RN35" that was discovered in September 2015 was determined. The diameter size estimates for this asteroid range from $79 \mathrm{~m}$ to $177 \mathrm{~m}$ and the mean value of $128 \mathrm{~m}$ was selected for the analysis. This asteroid has multiple impact instances over the next 100 years and the instance, that corresponds to a potential impact on Wednesday, October $20^{\text {th }} 2077$, was selected and the risk distribution is shown in Figure 13. The visualization shows those parts of the impact corridor that coincide with land masses as only direct land (and near coastal) impacts are considered.

Given the current impact probability of $3.06 \times 10^{-6}$, the risk associated with this impact is 0.0593 (worst case: 0.0797 ; best case: 0.0445 ). In comparison, the 
risk in the case of a hypothetical scenario of a certain impact (the integral of the global impact probability distribution equals one) would be 19419.043. (worst case: 26101.014 ; best case: 14575.453 ).

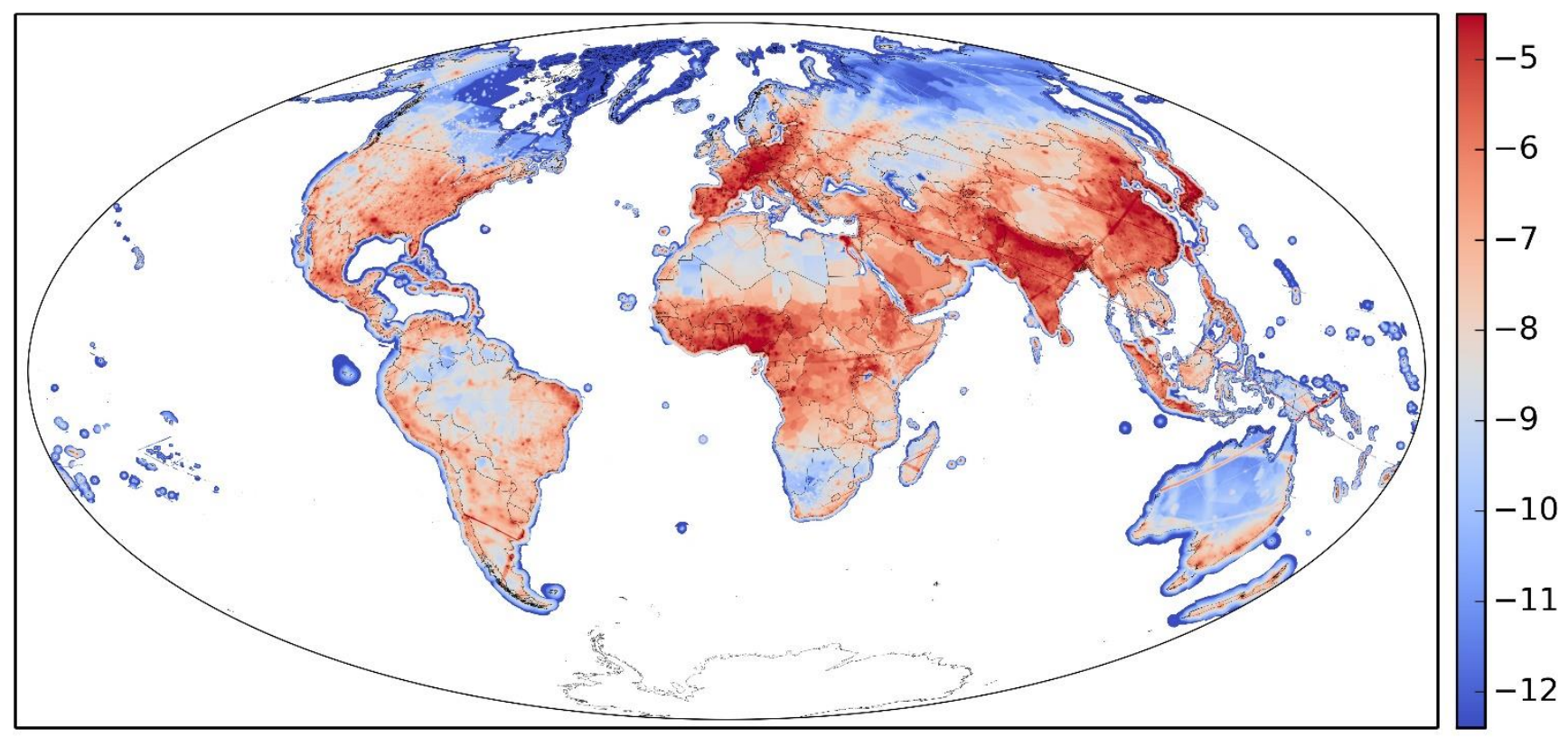

Figure 12: Global direct asteroid impact risk distribution in the expected case. Risk values are colour coded and are presented on a logarithmic scale with base ten.

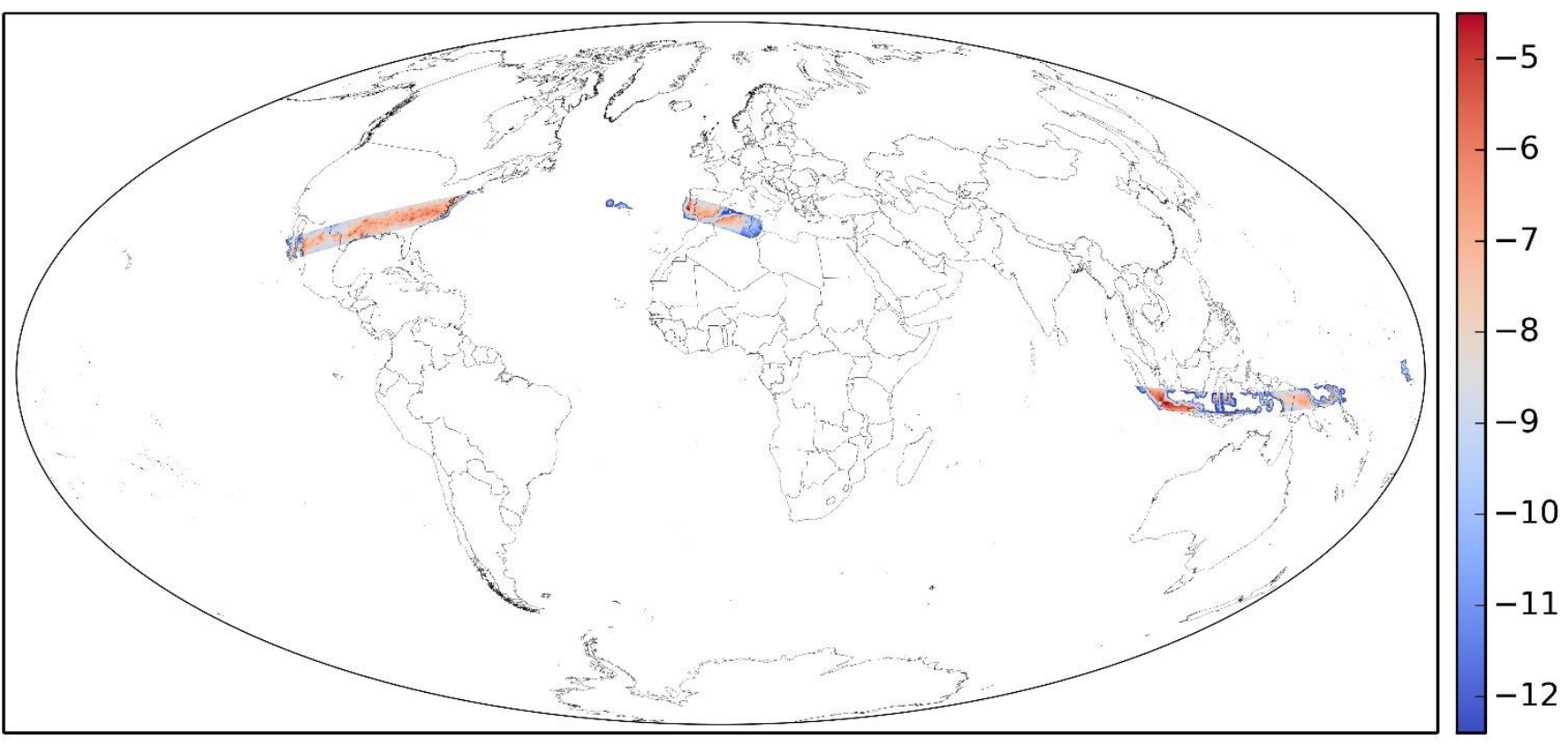

Figure 13: Direct impact risk corridor of the potential 2077/10/20 impact of asteroid 2015 RN35 


\section{DISCUSSION}

Risk calculations in this research are partly based on analytical models that are described in [17] and on vulnerability models that are described above. Both, impact effect and vulnerability models, are derived based on numerical simulation results in the first case and a combinatory literature review in the second case. Vulnerability models and their derivation have been explained in detail, on one hand, because the literature provided no coherent source for these data to date and, on the other hand, to facilitate reproduction of similar work. Given the rarity of impact events, it remains a challenge to validate impact effect as well as vulnerability models with recorded data, and, consequently, it is expected that the risk results are reasonable estimates but may be improved upon. At this time, the models enable further investigation of asteroid impact risk.

The global risk distribution of the 261 VI sample is shown in Figure 12 and this result serves to showcase the capabilities of ARMOR to calculate impact effects of known asteroids and the consequences for the population. After the definition of applicable models, the direct impact risks for the expected, best and worst case were determined. Considering that the impact probabilities of those potential impacts are low at the time of this writing, the total risk over the next 100 years is 13.59 casualties. Furthermore, the best case scenario is $24.3 \%$ lower than the expected case and the worst case increases risk by $33.8 \%$. The case deviations provide a sense for the variability in the risk values that is caused by modifications of the vulnerability models. One may observe that risk estimates remain well within one order of magnitude. This outcome could mean that vulnerability variation for best and worst case scenarios were given insufficient variability. However, the a priori designation of best/worst case variability was made with the intention to cover the expected range of vulnerability and the low sensitivity to case variations is a surprise. Additional research in vulnerability variability should be conducted but such research may require high fidelity numerical simulations of asteroid entries or, better, actual observational data, if available.

The risk of the sample is relatively low compared to what is expected for other natural disasters over a 100 years period. However, the data only offer limited comparability to these information because the sample does not account for the entire asteroid population - only about $1 \%$ of all NEOs are observed [35], and, thus, does not capture all asteroids that could impact in the next 100 years. Furthermore, the probabilities that are assigned to the sample VIs might change in the future as new observations for the included asteroids become available.
However, given that the sample impact probabilities suggest that none of these impacts will actually occur, the risk value appears to be plausible, small as it is.

At this point, it should be reiterated that the risk calculation did only account for direct land (and nearcoastal) impacts. It is expected that the inclusion of tsunamis will modify risk estimates significantly because tsunamis are thought to be a significant risk factor and this subject will be addressed in the future.

In the second part of the analysis, the risk of one specific asteroid, 2015 RN35, was analysed. Based on the observations that were available at the time of this writing, the impact probability of the selected impact instance of this asteroid was $3.06 \times 10^{-6}$, and, consequently, the projected risk is low with 0.0593 . Similarly to the sample analysis, best and worst case vulnerability cases were determined for $2015 \mathrm{RN} 35$ and risk varied by $25.0 \% /+34.4 \%$ for the best/worst case scenarios considering the current impact probability. In summary, the risk calculation, based on the single asteroid as well as on the sample, showed similar variability for the best and worst cases. The results suggest that the impact conditions determining variability for all asteroid risk case calculations were similar despite covering different parts of the planet or being assigned different impact probabilities. It could be that the potential impact location representation in the shape of impact probability distributions results in a generally similar coverage of ground-water masses.

To explore the unlikely but possible future scenario that the impact probability of this VI increases, an impact probability of one was assigned to the VI and risk increased to 19419.043. This risk demonstrates how serious the situation with this specific asteroid could potentially become. A similar analysis could be conducted for any observed asteroid on the risk list. It would thus be possible to classify asteroids according to their actual risk, taking into account their impact location, physical characteristics (size, impact angle and speed) and impact probability.

Two impact threat scales exist to date: The Torino scale [36] is intended as a risk communication device with the general public and the Palermo scale [37] provides a continuous reading that is better suited for expert interpretation. Both scales rely on impact kinetic energy as a proxy for the potential damage that a specific asteroid might cause and on the impact probability of this asteroid. The method presented here has the potential to form the basis of a new risk scale that estimates potential damage directly. This, and the effect of impact lead time in such a scale, will be addressed in future work. 


\section{CONCLUSIONS}

This paper introduces the impact effect and population vulnerability models that are used by ARMOR for asteroid land impact risk calculation. Using the software tools OrbFit and ARMOR allows to calculate risk estimates for observed asteroids taking into account impact location, impact probability and impact conditions such as asteroid size, speed and angle. Risk calculations are performed for a sample consisting of 261 potential future impact instances (VIs) of 69 asteroids until 2100 and for a single, recently discovered asteroid 2015 RN35.

In order to explore the sensitivity of the vulnerability models, best, expected and worst case scenarios are defined and evaluated. The resulting risk variability remains well within one order of magnitude which could mean that the assumptions for best/worst cases were not spread wide enough and that the topic of vulnerability model variability deserves further attention.

The risk of the sample was 13.59 casualties for the next 100 years and the low risk result is sensible considering that the impact probabilities of the sample asteroids are small and an actual impact of those asteroids is not expected.

Additionally, the risk of recently discovered asteroid 2015 RN35 was calculated as 0.0593 based on a low impact probability of $3.06 \times 10^{-6}$. To explore how serious this threat could become in the event of increasing impact probability, a unitary impact probability was assigned and the resulting risk to direct land (or near-coastal) impacts increased to 19419.043 casualties.

ARMOR's potential to form the basis of a new asteroid risk scale will be explored in the future.

\section{ACKNOWLEDGMENT}

Peter M. Atkinson is grateful to the University of Utrecht for supporting him with The Belle van Zuylen Chair. The work is supported by the Marie Curie Initial Training Network Stardust, FP7-PEOPLE-2012-ITN, Grant Agreement 317185.

\section{BIBLIOGRAPHY}

[1] G. W. Wetherill, "Formation of the Earth," Аnпи. Rev. Earth Planet. Sci., vol. 18, pp. 205-256, 1990.
G. Ryder, "Mass flux in the ancient Earth-Moon system and benign implications for the origin of life on Earth," J. Geophys. Res., vol. 107, no. E4, 2002.

[3] L. W. Alvarez, W. Alvarez, F. Asaro, et al., "Extraterrestrial cause for the cretaceous-tertiary extinction.," Science (80-. )., vol. 208, no. 4448, pp. 1095-1108, 1980.

[4] O. P. Popova, P. Jenniskens, V. Emel'yanenko, et al., "Chelyabinsk airburst, damage assessment, meteorite recovery, and characterization.," Science, vol. 342, no. 6162, pp. 1069-73, Nov. 2013.

[5] C. F. Chyba, P. J. Thomas, and K. J. Zahnle, "The 1908 Tunguska explosion: atmospheric disruption of a stony asteroid," Nature, vol. 361, no. January, 1993.

[6] National Research Council, I. I. Shapiro, F. Vilas, et al., "Defending Planet Earth : Near-Earth Object Surveys and Hazard Mitigation Strategies," Washington D.C., 2010.

[7] Universita Di Pisa and European Space Agency, “NEODyS-2 Risk Page," 2014. [Online].

Available: http://newton.dm.unipi.it/neodys/index.php?pc=4 . 1.

[8] NASA, "NASA NEO Impact Risk," 2014. [Online]. Available: http://neo.jpl.nasa.gov/risk/.

[9] A. Milani, G. F. Gronchi, G. Tommei, et al., "The OrbFit Software Package," GNU General Public License, 1997. [Online]. Available: http://adams.dm.unipi.it/orbfit/. [Accessed: 08Jan-2015].

[10] C. Rumpf, H. G. Lewis, and P. M. Atkinson, "MONITORING THE GLOBAL ASTEROID IMPACT RISK," in 66th IAF International Astronautical Congress, 2015, vol. IAC-15.B5.

[11] CIESIN, Columbia University, United Nations Food and Agriculture Programme - FAO, et al., "Gridded Population of the World, Version 3 (GPWv3): Population Count Grid, Future Estimates," NASA Socioeconomic Data and Applications Center (SEDAC), Palisades, NY, 2005.

[12] C. Rumpf, H. G. Lewis, and P. M. Atkinson, "GLOBAL IMPACT DISTRIBUTION OF ASTEROIDS AND AFFECTED POPULATION," in IAA Planetary Defense Conference, 2015, no. 4th. 
[13] N. E. Klepeis, W. C. Nelson, W. R. Ott, et al., "The National Human Activity Pattern Survey (NHAPS): a resource for assessing exposure to environmental pollutants," J. Expo. Anal.

Environ. Epidemiol., vol. 11, no. 3, pp. 231-252, 2001.

[14] B. L. Diffey, "An overview analysis of the time people spend outdoors," Br. J. Dermatol., vol. 164, no. 4, pp. 848-854, 2011.

[15] Trades Union Congress, "Average Commuting Times in UK," Labour Force Survey, 2012. [Online]. Available: https://www.tuc.org.uk/workplace-issues/worklife-balance/men-their-early-40s-have-longestcommutes. [Accessed: 21-Jul-2015].

[16] U.S. Census Bureau, "Average Commute Times in the United States," U.S. Census American Community Survey 5-year estimates, 2011. [Online]. Available: http://project.wnyc.org/commute-timesus/embed.html\#5.00/36.114/-92.526. [Accessed: 21-Jul-2015].

[17] G. S. Collins, H. J. Melosh, and R. a. Marcus, "Earth Impact Effects Program: A Web-based computer program for calculating the regional environmental consequences of a meteoroid impact on Earth," Meteorit. Planet. Sci., vol. 40, no. 6, pp. 817-840, Jun. 2005.

[18] D. Britt, "The Physical Properties of Near Earth Asteroids," in steroid Grand Challenge: Virtual Seminar Series, 2014, pp. 1-30.

[19] S. Glasstone and P. J. Dolan, The Effects of Nuclear Weapons, Third Edit. USA: United States Department of Defense \& Energy Research and Development Administration, 1977.

[20] Wind Science and Engineering Center, “A Recommendation for an Enhanced Fujita Scale (EF-Scale)," Lubbock, Texas, 2006.

[21] NOAA, "NOAA's National Weather Service, Storm Prediction Center, Killer Tornados by Year," Data on Webpage, 2015. [Online]. Available:

http://www.spc.noaa.gov/climo/historical.html. [Accessed: 28-Apr-2015].

[22] Y. B. Zel'dovich and Y. P. Raizer, Physics of Shock Waves and High-Temperature Hydrodynamic Phenomena. New York: Academic Press, 1966.
[23] I. V. Nemtchinov, V. V. Shuvalov, N. a. Artemieva, et al., "Light flashes caused by meteroid impacts on the lunar surface," Sol. Syst. Res., no. 32, pp. 99-114, 1998.

[24] J. Ortiz, P. Sada, L. R. Bellot Rubio, et al., "Optical detection of meteoroidal impacts on the Moon," Nature, vol. 405, no. 6789, pp. 921-3, 2000.

[25] I. V. Nemtchinov, O. P. Popova, V. V. Shuvalov, et al., "Radiation emitted during the flight of asteroids and comets through the atmosphere," Planet. Space Sci., vol. 42, no. 6, pp. 491-506, 1994.

[26] J. Borovička and P. Kalenda, "The Morávka meteorite fall: 4. Meteoroid dynamics and fragmentation in the atmosphere," Meteorit. Planet. Sci., vol. 38, no. 7, pp. 1023-1043, 2003.

[27] American Burn Association, "National Burn Repository Report of Data From 2002-2011," Chicago, IL, 2012.

[28] P. H. Schultz and D. E. Gault, "Seismic Effects From Major Basin Formations on the Moon and Mercury," Moon, vol. 12, pp. 159-177, 1975.

[29] C. Norlund, H. G. Lewis, and P. M. Atkinson, "Evacuation Simulation Modelling in the Event of a Near Earth Object impact," in First International Conference on Evacuation Modeling and Management, 2009, p. 10.

[30] S. Wu, J. Jin, and T. Pan, "Empirical seismic vulnerability curve for mortality: case study of China," Nat. Hazards, vol. 77, no. 2, pp. 645-662, 2015.

[31] US Geological Survey, "The Severity of an Earthquake," Website, 2015. [Online]. Available: http://pubs.usgs.gov/gip/earthq4/severitygip.html. [Accessed: 24-Aug-2015].

[32] C. Norlund, "Evacuation Simulation Modelling in the event of a Near Earth Object impact," University of Southampton, 2013.

[33] A. Pomonis, R. Spence, and P. Baxter, "Risk assessment of residential buildings for an eruption of Furnas Volcano, Sao Miguel, the Azores," J. Volcanol. Geotherm. Res., vol. 92, no. 1-2, pp. 107-131, 1999.

[34] C. Rumpf, H. G. Lewis, and P. M. Atkinson, "The global impact distribution of Near-Earth objects," Icarus, vol. 265, pp. 209-217, 2016. 
[35] A. W. Harris and G. D'Abramo, "The population of near-earth asteroids," Icarus, no. May, 2015.

[36] R. P. Binzel, "The Torino Impact Hazard Scale," Planet. Space Sci., vol. 48, no. 4, pp. 297-303, 2000.

[37] S. R. Chesley, P. W. Chodas, A. Milani, et al., "Quantifying the Risk Posed by Potential Earth Impacts," Icarus, vol. 159, no. 2, pp. 423-432, Oct. 2002.

\section{BIOGRAPHY}

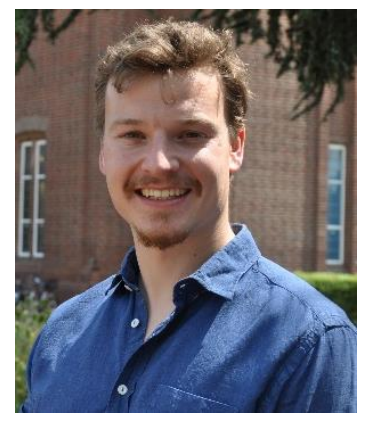

In 2012, Clemens Rumpf

received his "Diplom-

Ingenieur" aerospace

engineering degree from

Braunschweig University of

Technology in Germany and

Purdue University in the

United States. The topic of his

diploma thesis was the design

of a navigation filter for a vertical take-off and landing vehicle at the DLR Institute of Space Systems in Bremen. Working as a GNC and space system engineer, he was part of the ESA Lunar Lander team at ESTEC in the Netherlands between 2012 and 2013. Since 2013, he is a member of the European Stardust research network and pursues a $\mathrm{PhD}$ in asteroid impact risk assessment at the University of Southampton in the United Kingdom where he also initiated and leads a project to build the University's first CubeSat.

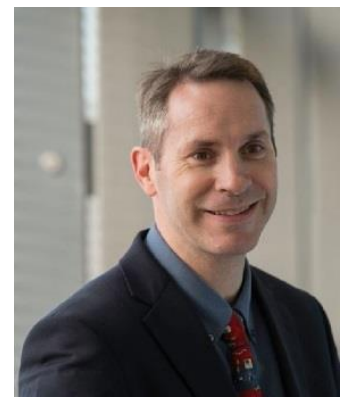

Hugh G. Lewis gained a Master's degree in Control Systems from the University of Sheffield in 1993 and a PhD in Remote Sensing from the University of Southampton in 1998.

Today, he is a Senior Lecturer in Aerospace Engineering at the University of Southampton, author of the space debris evolutionary model DAMAGE and leads the Astronautics Research Group's space debris and space situational awareness activities. He is a member of the UK Space Agency delegation to the Inter-Agency Space Debris Coordination Committee (IADC) and the Space Missions Planning
Advisory Group (SMPAG), and he is also a member of the UK delegation to the United Nations Committee on the Peaceful Uses of Outer Space (UN COPUOS).

Dr Lewis primary research interests are space situational awareness relating to space debris and the NEO hazard and ways to meet these challenges. He is also interested in artificial neural networks and their application in fields such as remote sensing, fractionated satellite concepts and psychology.

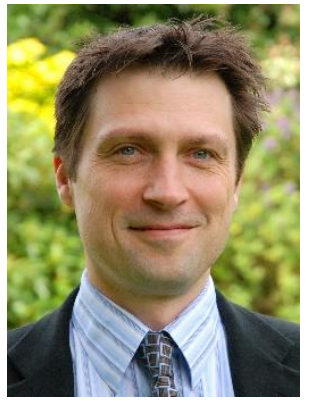

Peter M. Atkinson received the BSc degree in Geography from the University of Nottingham in 1986 and the $\mathrm{PhD}$ degree from the University of Sheffield (NERC CASE award with Rothamsted Experimental Station) in 1990. More recently, he received the MBA degree from the University

of Southampton in 2012.

$\mathrm{He}$ is currently the Dean of the Faculty of Science and Technology at Lancaster University. $\mathrm{He}$ has been Professor of Geography at the University Southampton (for the last 21 years; 13 as Professor), where he is currently Visiting Professor. He is also currently holder of the Belle van Zuylen Chair in the Faculty of Geosciences at Utrecht University, the Netherlands, and Visiting Professor at Queen's University Belfast, UK. He was recently (2012-14) Visiting Fellow at Green-Templeton College and the Department of Zoology, Oxford University.

The main focus of his research is in remote sensing, geographical information science and spatial (and spacetime) statistics applied to a range of environmental science and socio-economic problems. Prof. Atkinson has published around 200 peer-reviewed articles in international scientific journals. Prof. Atkinson is Associate Editor for Computers and Geosciences and sits on the editorial boards of several further journals including Geographical Analysis, Spatial Statistics, the International Journal of Applied Earth Observation and Geoinformation, and Environmental Informatics. He sits on various international scientific committees. 